\title{
TOOTH FORM ASSESSMENT IN THE AESTHETIC ZONE OF THE MAXILLA
}

\author{
Irena Georgieva \\ Department of Periodontology and Dental Implantology, Faculty of Dental Medicine, \\ Medical University of Varna
}

\begin{abstract}
INTRODUCTION: The accurate assessment of tooth form and gingival phenotype in the aesthetic zone of maxilla is of great importance for the correct preoperative planning in dental practice. Determining the tooth form as a key factor helps the clinicians in assessing the aesthetic risk in periodontal, restorative and implant therapy.

AIM: The aim of this study is to investigate the prevalence of rectangular and triangular tooth form in the aesthetic zone of the maxilla and also to generalize the variations in this prevalence between males and females in the Bulgarian population.
\end{abstract}

MATERIALS AND METHODS: The tooth form in the aesthetic zone of maxilla is assessed in 223 individuals and determined according to four anatomic criteria: width and position of the interdental contact point, CW/CL ratio, height of interdental papillae, and the scallop of gingival margin.

RESULTS: The analysis of the results shows that the rectangular tooth form is more prevalent (70\%) compared to the triangular tooth form (30\%), and the triangular tooth form is more prevalent in males (32\%) than in females (29\%).

DISCUSSION: With regard to the tooth form in approximately $70 \%$ of the cases (prevalence of a square/rectangular tooth form) we can expect to have a lower aesthetic risk in the preoperative planning of implant therapy in the aesthetic zone of the maxilla.

CONCLUSION: More prevalent in the study group is the rectangular/square tooth form (70\%) compared to the triangular tooth form $(30 \%)$ with a ratio of $2.33: 1$.

Keywords: tooth form, maxilla, aesthetic zone, aesthetic risk, front teeth

\section{Address for correspondence: \\ Irena Georgieva \\ Faculty of Dental Medicine \\ Medical University of Varna \\ 84 Tzar Osvoboditel Blvd \\ 9000 Varna \\ e-mail:dr.irenageorgieva@mail.bg}

Received: November 26, 2019

Accepted: December 4, 2019

\section{INTRODUCTION}

In the aesthetic zone of maxilla, the periodontal and implant therapy requires accurate and correct preoperative planning and precise clinical assessment of all key factors that are important for the successful final aesthetic results of the dental treatment. The predictability of the post-treatment results of periodontal and implant therapy is better after precise clinical and radiographic assessment. The 
aesthetic risk is highly dependent on the thickness of gingival tissues and the tooth form of the crowns of upper front teeth. That is why the accurate assessment of the tooth form and gingival phenotype is important for the correct preoperative planning, which helps the clinicians to reduce the aesthetic risk.

The tooth form of dental crowns is a key consideration in periodontal, restorative and implant therapy.

In the beginning of 20th century, the form of the tooth crowns was classified in respect to the shape of the facial contour, but nowadays the classification of the form of tooth crowns divides the teeth on the basis of a different geometric shape - triangular, rectangular, and oval (1).

According to some clinical investigations, the most prevalent is the oval tooth form (47.06\%), after that is the rectangular one (31.37\%), and the least common is the triangular tooth form (27.57\%) (2).

Olsson and Lindhe (1991) use the ratio of coronal width (CW) and coronal length (CL) of central incisor crowns to determine them as long and narrow crowns when CW/CL \pm 0.5 , and as short and wide when CW/CL \pm 1.0 . There is a statistically significant difference between the CW/CL ratio of central maxillary incisors in male and female individuals (3). Both authors conclude that the CW/CL ratio is related to the thickness of the periodontal phenotype, the probing depth, and the occurrence of gingival recessions on the buccal surface of the tooth crowns $(3,4)$.

An CW/CL ratio of $80 \%$ is considered an ideal proportion for maxillary central incisors (5). Other authors report a coefficient of $75-80 \%$ in their researches (6). Thus, in the central upper incisors, an average CW/CL ratio of $0.81 \mathrm{~mm}$ was established (7).

In another study, Cook et al. (2011) modified the definition of the shape of tooth crowns by adding the criterion of contact point surface (CS) between adjacent teeth. Cook uses it to classify the form of tooth crowns as triangular and rectangular (8).

Another classification divides the form of tooth crowns into only triangular and rectangular ones. One study of Stellini et al. focused on the assessment of tooth form in males and females reported that $81 \%$ of all assessed males have rectangular form of tooth crowns and the other $19 \%$ - a triangular one, while
$84 \%$ of the assessed females have rectangular form of tooth crowns and the remaining $16 \%$ - a triangular one (9).

The rectangular shape of tooth crowns is associated with wide interdental contacts in the middle third of the interproximal surfaces of the adjacent teeth, wide and short interdental papillae, and thick periodontal phenotype. On the other hand, triangular tooth crowns have point-like contacts in the incisal third of the interproximal surfaces of the crowns of adjacent teeth, long and thin interdental papillae, and thin periodontal phenotype $(2,9)$.

Teeth with a rectangular form of crowns are associated with thin interdental alveolar bone and most prevalent developing vertical bone loss (10). Respectively, teeth with a triangular form of the crowns have wider and higher interproximal alveolar bone that is more rarely associated with vertical bone loss but more frequently with horizontal bone loss of interdental bone and increased risk of interdental papillae height loss and the appearance of the so-called "black triangles" and development of gingival recessions (9).

Achieving a harmonious outline of the gingival margin and intact interdental papillae depends to some extent on the form of the crowns of the upper front teeth (11).

The tooth form is one of the five essential key factors for periodontal and peri-implant aesthetics. The tooth form is important for the morphology of peri-implant soft tissues in surgical implant therapy and for the results of subsequent conventional and surgical periodontal manipulations. The tooth form is of great importance for the aesthetic risk and should be taken into consideration in the preoperative planning of regenerative periodontal and implant therapy (10).

\section{AIM}

The aim of this epidemiologic study is to investigate the prevalence of rectangular and triangular tooth form in the aesthetic zone of maxilla and also to generalize the variations in this prevalence between males and females in the Bulgarian population. It tries to determine the significance of these variations regarding the aesthetic risk in implant therapy and periodontal surgical therapy in the aesthetic zone. 


\section{MATERIALS AND METHODS}

The study includes 223 individuals (81 males and 142 females) between 18 and 75 years of age mean age 37.37 years. The sex distribution is $64 \% \mathrm{fe}-$ males and $36 \%$ males.

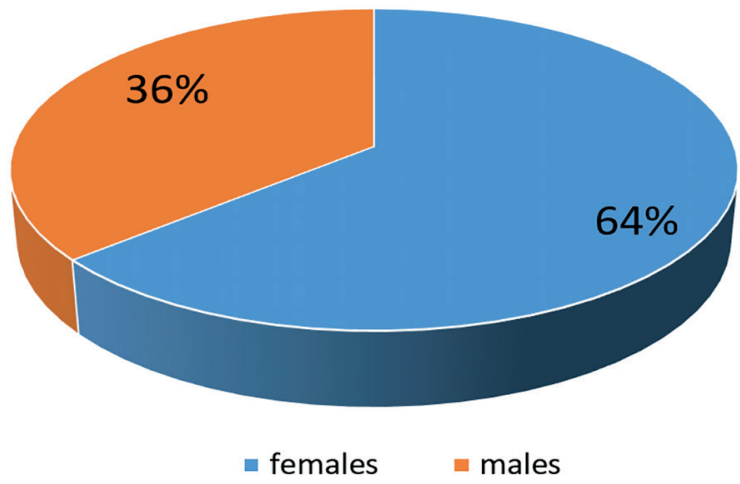

Fig. 1. Sex distribution of examined individuals

The test group includes only patients, who respond to the following criteria:

$\diamond$ all individuals are over 18 years of age;

$\diamond$ all participants in the study have permanent dentition in the frontal sextant of the maxilla.

Potential subjects who meet any of the following criteria were excluded from participating in the study:

$\diamond$ patients with temporary upper front teeth;

$\diamond$ patients with prosthetic constructions of the upper front teeth;

$\diamond$ patients with conservative restored upper front teeth leading to change in the form of tooth crowns;

$\diamond$ patients with missing maxillary front teeth;

$\diamond$ patients with abrasion of the upper front teeth.

In all individuals in the test group, the tooth form in the aesthetic zone of the maxillae, is clinically assessed and determined according to four anatomic criteria: width and position of the interdental contact point, CW/CL ratio, height of interden- tal papillae, and the scallop of the gingival margin Based on these criteria, the teeth are divided into 2 groups: teeth with a rectangular (square) form and teeth with a triangular form. The ovoid form of the tooth crowns is not considered in this study.

Statistical analysis of the results is done applying a descriptive analysis using IBM SPSS Statistics software version 19.

\section{RESULTS}

In the assessment of all 223 males and females, we received the following results:

$\diamond 156$ of the assessed patients have a rectangular/ square form of the tooth crowns of the upper front teeth - 70\% of all individuals;

$\diamond 67$ of the assessed patients have a triangular form $(30 \%)$.

The received data of the conducted study are generalized in Table 1 and on Fig. 2:

Relative to the sex of the assessed patients, we registered the following results of the tooth form in the frontal sextant of the upper jaw:

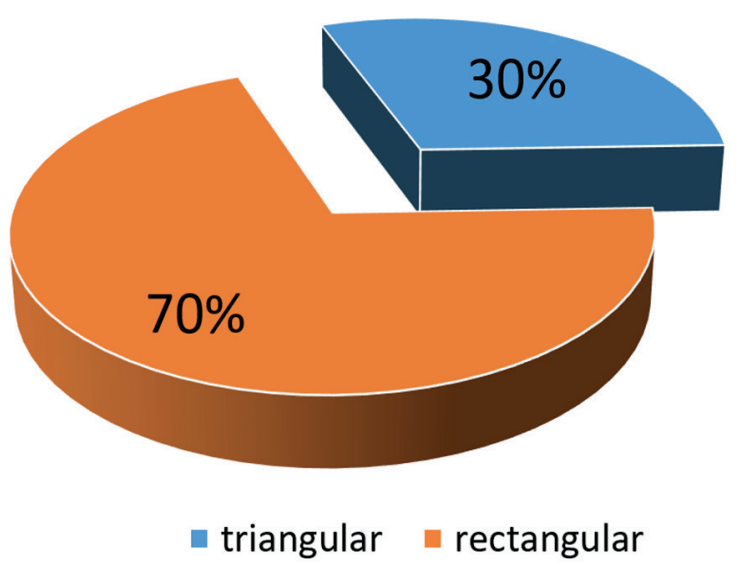

Fig. 2. Distribution of patients of both sexes according to the tooth form of the crowns in the aesthetic zone of the maxilla (in percentages)

Table 1. Distribution of the tooth form in Bulgarian population

\begin{tabular}{l|c|c|c|c|c|c|}
\hline \multirow{2}{*}{ Tooth Form } & Males & Males & Females & Females & Total & Total \\
\cline { 2 - 7 } & $(\mathrm{n})$ & $\%$ & $(\mathrm{n})$ & $\%$ & $(\mathrm{n})$ & $\%$ \\
Triangular form & 26 & 32 & 41 & 29 & 66 & 30 \\
Rectangular form & 55 & 68 & 101 & 71 & 154 & 70 \\
\hline \hline
\end{tabular}


\& 81 males - 26 males with a triangular form (32\%) and 55 males with a rectangular form (68\%) (Fig. 3);

$\diamond 142$ females -41 females with a triangular tooth form (29\%) and 101 females with a rectangular form (71\%) (Fig. 4).

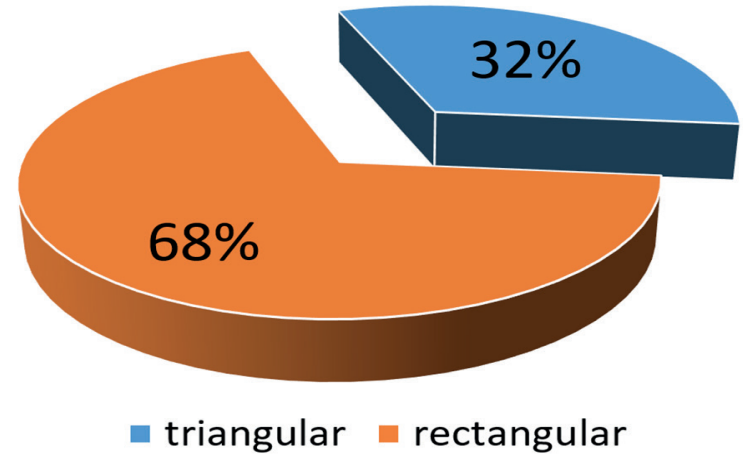

Fig. 3. Distribution of patients according to the tooth form of the crowns in the aesthetic zone of maxilla (in males)

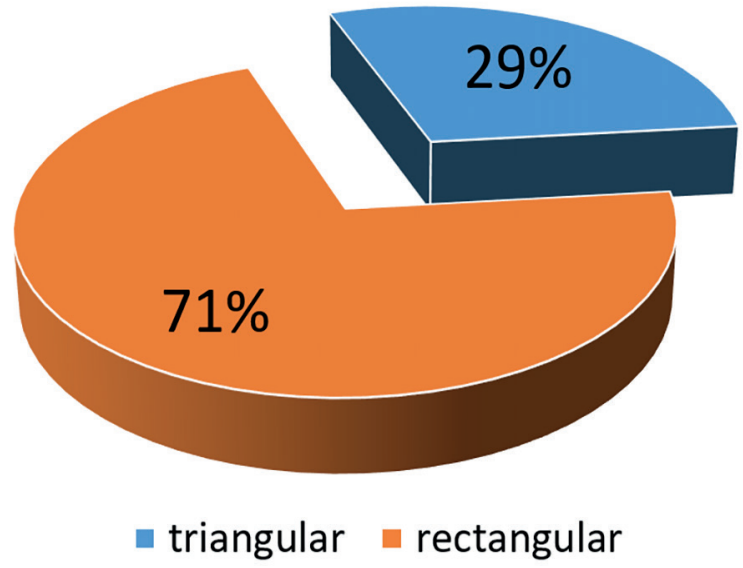

Fig. 4. Distribution of patients according to the tooth form of the crowns in the aesthetic zone of maxilla (in females)

\section{DISCUSSION}

The analysis of the received results in this study reveals that in relation to the sex we can conclude that in male participants the triangular form of the tooth crowns is more prevalent (32\%) than in female individuals (29\%) - with a ratio 1.1:1.

Respectively, the rectangular/square form of the tooth crowns is more prevalent in females (71\%) than in males (68\%) - with a ratio 1:1.1. (Fig. 3 and Fig. 4)
The received results in the assessed patients from the Bulgarian population do not correspond to the results in the study of Stellini et al. In their study, Stellini et al. report that the rectangular/square form of tooth crowns is prevalent in $81 \%$ of all males in the test group, and the triangular form is prevalent only in the remaining $19 \%$ of the participants. They also report that $84 \%$ of the assessed females have a rectangular/square tooth form, and the remaining $16 \%$ of the women in the test group have a triangular form (9).

The results of our research, however, confirm those obtained in the study by Stellini et al. that the triangular form of the tooth crowns is slightly more prevalent in male representatives (9).

The summarized results of all 223 assessed Bulgarian individuals in this study show that a prevalence of a triangular form of the upper front teeth is established in $30 \%$ of participants and a rectangular/ square form - in $70 \%$ of them (1:2.33) (Table 1).

Individuals with a square/rectangular tooth form are more suitable for implant therapy. In this case, a better aesthetic result and lower aesthetic risk is predicted for the preoperative planning due to the wider contact surfaces (10). Individuals with a triangular form of tooth crowns, especially in combination with localized periodontal defects and loss of interproximal papillae, are less suitable for surgical periodontal and implant therapy due to higher aesthetic risk in the aesthetic zone of the maxilla $(12,13)$.

According to the results of the recent study, in approximately $70 \%$ of the cases (prevalence of a square/rectangular tooth form) we can expect to have a lower aesthetic risk in the preoperative planning of implant therapy in the aesthetic zone of the maxilla. In the remaining $30 \%$ of cases (triangular tooth form), there will be a higher preoperative aesthetic risk, and we can expect difficulties and unsatisfactory healing results in conventional and surgical periodontal therapy, and implant treatment in the aesthetic zone of the maxilla.

After analyzing the results obtained in this study, we can conclude that the sex of individuals is not of such high importance in predicting and assessing the aesthetic risk in preoperative planning in the aesthetic zone of the maxilla, but we can conclude also that the aesthetic risk in preoperative planning 
will be higher in males because of the more prevalent triangular tooth form among them.

\section{CONCLUSION}

More prevalent in the study group is the rectangular/square tooth form (70\%) compared to the triangular tooth form (30\%) with a ratio of $2.33: 1$. The triangular tooth form is more prevalent in male individuals - $32 \%$ of cases, than in females - $29 \%$ of assessed women.

\section{REFERENCES}

1. Sambhav J, Munish R, Pradeep R, Shalu J, Arbab A, Vaibhav M, Ragini S. Assessment of tooth proportions in an aesthetically acceptable smile. J Clin Diagn Res. 2015;9(4):ZC01-4. doi: 10.7860/ JCDR/2015/11338.5771.

2. McGowan S. Characteristics of teeth: a review of size, shape, composition, and appearance of maxillary anterior teeth. Compend Contin Educ Dent. 2016;37(3):164-171;quiz172.

3. Olsson $\mathrm{M}$, Lindhe J. Periodontal characteristics in individuals with varying form of the upper central incisors. J Clin Periodontol. 1991;18(1):78-82. doi: 10.1111/j.1600-051x.1991.tb01124.x.

4. Claffey N, Shanley D. Relationship of gingival thickness and bleeding to loss of probing attachment in shallow sites following nonsurgical periodontal therapy. J Clin Periodontol. 1986;13(7):654-7. doi: 10.1111/j.1600-051x.1986. tb00861.x.

5. Gehrke R, Strohecker A, Dhom G. Influence of interdental papilla length and interproximal contact point on the perception of esthetics in symmetric and asymmetric situations. J Dent Implantol. 2010; 3:230-46. doi: 10.3238/ ZZI.2010.0230.
6. Chiche GJ, Pinault A. Esthetics of anterior fixed prosthodontics. Chicago: Quintessence; 1993.

7. Lyssova V, Estafan D, Cunnigham RP. A multidisciplinary esthetic approach to single-tooth replacement and diastema closure. Gen Dent. 2008;56(3):282-5.

8. Jon L, Morante D, Bernabé E, Vich M, Cotrina L. Esthetic perception towards different combinations of facial contours and upper incisor shape. Braz J Oral Sci. 2009; 8(4):193-6.

9. Stellini E, Comuzzi L, Mazzocco F, Parente N, Gobbato L. Relationships between different tooth shapes and patient's periodontal phenotype. J Periodontal Res. 2013 Oct;48(5):657-62. doi: 10.1111/jre.12057.

10. Liébert MF, Fouque-Deruelle C, Santini A, Dillier FL, Monnet-Corti V, et al. Smile line and periodontium visibility. Periodont Practice Today. 2004; 1(1):17-25.

11. Sharma A, Park JH. Esthetic considerations in interdental papilla: remediation and regeneration. J Esthet Restor Dent. 2010 Feb;22(1):18-28. doi: 10.1111/j.1708-8240.2009.00307.x.

12. Ahmad I. Anterior dental aesthetics: Gingival perspective. Br Dent J. 2005;199(4):195-202.

13. Singh VP, Uppoor AS, Nayak DG, Shah D. Black triangle dilemma and its management in esthetic dentistry. Dent Res J (Isfahan). 2013;10(3):296-301 